\title{
Predictive Factors for Recovery Time in Conceived Women Suffering From Moderate to Severe Ovarian Hyperstimulation Syndrome
}

\section{Kai Huang}

Center for Reproductive Medicine, The First Affiliated Hospital of Zhengzhou University, Zhengzhou

\section{Ying Shi}

Center for Reproductive Medicine, The First Affiliated Hospital of Zhengzhou University, Zhengzhou

\section{Gezi Chen}

Department of Obstetrics, The First Affiliated Hospital of Zhengzhou University, Zhengzhou

\section{Hao Shi}

Center for Reproductive Medicine, The First Affiliated Hospital of Zhengzhou University, Zhengzhou Jun Zhai ( $\nabla$ bestzhai2005@163.com )

Center for Reproductive Medicine, The First Affiliated Hospital of Zhengzhou University, Zhengzhou

\section{Research Article}

Keywords: ovarian hyperstimulation syndrome (OHSS), Clinical features, Recovery time, Potential predictors

Posted Date: January 28th, 2022

DOI: https://doi.org/10.21203/rs.3.rs-1292102/v1

License: (c) (i) This work is licensed under a Creative Commons Attribution 4.0 International License. Read Full License 


\section{Abstract}

Backgroud: To evaluate potential predictors for recovery time in pregnant patients with moderate to severe ovarian hyperstimulation syndrome.

Methods: A total of 424 pregnant patients with moderate to severe OHSS underwent in vitro fertilization/intracytoplasmic sperm injection (IVF/ICSI) were retrospectively identified. The clinical features and laboratory findings within 24 hours after admission were collected. Treatment of OHSS was carried out according to standard procedure including fluid replacement therapy, human albumin, aspirin, low molecular weight heparin and paracentesis if necessary. Patients were discharged from hospital when morning hematocrit was $<40 \%$ and no obvious clinically relevant symptoms were existed, such as abdominal distension, abdominal pain and shortness of breath. Meanwhile, ultrasound indicated little pleural or abdominal effusion and biochemical abnormalities return to normal were required.

Results: The median recovery time of these patients was 11 days. In spearman correlation test, leukocyte, hemoglobin, platelets, hematocrit, creatinine, PT, Fib, D-dimer, and FDP were positively correlated with recovery time. Whereas, albumin and TT were negatively correlated with recovery time. Multiple linear regression analysis showed that polycystic ovary syndrome (PCOS), hemoglobin, platelets, albumin, and Fib significantly associated with the recovery time of OHSS patients $(p=0.023, p<0.001, p=0.007, p<$ 0.001 , and $p=0.019$; respectively).

Conclusions: In pregnant OHSS patients, presence of PCOS and hypoalbuminemia were associated with significantly longer recovery time. Meanwhile, recovery time was longer when patients have high levels of hemoglobin, platelets and Fib.

\section{Introduction}

Ovarian hyperstimulation syndrome (OHSS) is a self-limiting disease, classically encountered in patients who undergo controlled ovarian hyperstimulation $(\mathrm{COH})$ cycles. The mild manifestations of OHSS are nausea, vomiting, abdominal distension and shortness of breath. In severe cases, ascites and pleural fluid may occur, causing respiratory, circulatory and coagulation dysfunction, and especially thrombosis may endanger the patient's life [1].

Evidence from initial investigations indicates that some women are at increased risk for OHSS. These risk factors include young age [2,3], body mass index (BMI) [4,5], diagnosis of polycystic ovary syndrome (PCOS) $[3,6]$ high antimüllerian hormone $(\mathrm{AMH})$ level $[7,8]$, large number and size of follicles in the ovary [8,9], high serum E2 concentrations [3,9,10] high number of retrieved oocytes [3,9,11], pregnancy following fresh embryo transfer [12], and a history of OHSS [13]. The exact cause of OHSS is currently complex and remains subject to controversy. Latest research has demonstrated that OHSS is related to age, BMI, ovarian function, and the ovulation stimulation protocol. 
Evidence has shown that OHSS occurs only after exposure to human chorionic gonadotropin (hCG), which has a significantly longer half-life than LH and a higher receptor affinity, and thus causes extensive luteinization in the granulosa cells within the corpus luteum [14]. This in turn leads to production of vasoactive substances, including vascular endothelial growth factor (VEGF), renin-angiotensin system, interleukin-6, interleukin-1b, angiotensin II, insulin-like growth factor 1 , transforming growth factor $b$, of which VEGF is the most important in causing increased vascular permeability and hemoconcentration [15-17]. VEGF stimulates endothelial cell mitogenesis and renders capillaries highly permeable to high-molecular-weight proteins [15]. The pathophysiology of OHSS is characterized by arteriolar vasodilation and an increase in capillary permeability, leading to leakage of fluid from the vascular compartment, with third space fluid accumulation and intravascular dehydration, causing intravascular volume depletion, haemoconcentration, hypoalbuminaemia, electrolyte imbalance, and even thrombosis.

Whereas treatment for OHSS is largely supportive, prevention is crucial. Most current studies are devoted to prevention and treatment strategies for OHSS, with relatively little attention paid to the clinical prognosis of OHSS. This study provides clinicians with potential predictors of time to cure by describing some clinical features and laboratory findings in pregnant patients with OHSS.

\section{Materials And Methods}

Study Population

This retrospective study was performed in the Reproductive Medical Center of First Affiliated Hospital of Zhengzhou University, Henan Province, China. Patients who underwent in vitro fertilization/intracytoplasmic single sperm injection (IVF/ICSI) after assisted conception with late onset moderate to severe OHSS between January 2018 and December 2020 were selected. The access and processing of patient data was approved by the ethics committee under a protocol for retrospective studies. Inclusion criteria were as follows: 1) diagnosis of OHSS according to the Golan criteria; 2) Patients with IVF/ICSI assisted pregnancy in the first cycle; 3 ) have a positive pregnancy test; and 4) age $<35$ years. Exclusion criteria included: 1) Women treated with antithrombotic drugs and women with known coagulopathies; 2) Uncertain laboratory results and missing laboratory data. To analyze the relationship between the recovery time of OHSS patients and the blood parameters such as leukocyte, hemoglobin, platelet, hematocrit, creatinine, total protein, albumin, prothrombin time (PT), activated partial thromboplastin time (APTT), thrombin time (TT), fibrinogen (FIB), d-dimer and fibrinogen degradation products (FDP) within 24 hours after admission.

Treatment of OHSS usually involves fluid replacement to maintain intravascular perfusion and supportive care, such as low molecular dextrose and hydroxyethyl starch. The patient's blood count, coagulation profile, electrolytes, creatinine, and albumin were observed. Depending on the patient's condition, albumin was given intravenously, and anticoagulant drugs are given to patients with thrombotic tendency and hypercoagulable state to prevent thrombosis [18]. Moreover, if the patient has a large amount of pleural 
and ascites, puncture and drainage will be performed under the guidance of ultrasound. The details of the patient's treatment are shown in Table 1.

The patient is clinically cured when morning hematocrit was $<40 \%$ and no obvious clinically relevant symptoms were existed, such as abdominal distension and abdominal pain, shortness of breath [19]. Meanwhile, ultrasound indicated no pleural and abdominal effusion or a small amount of effusion, leucocyte count, creatinine, albumin, Alanine transaminase $\triangle A L T \rrbracket$, aspartate transaminase $\mathbb{A S T} \bigotimes$, electrolyte and other biochemical indicators return to normal. The discharge criteria were the patients' cure criteria.

Table 1 conventional intervention of OHSS

\begin{tabular}{|lll|}
\hline Treatment & $\mathrm{n}$ & $\%$ \\
\hline heparin & 46 & 10.8 \\
\hline albumin & 321 & 75.7 \\
\hline paracentesis & & \\
\hline Peritoneal puncture & 219 & 51.7 \\
\hline pleural puncture & 52 & 12.3 \\
\hline
\end{tabular}

\section{Laboratory Variables}

The patients underwent basic blood routine, liver and kidney function, blood coagulation function, Ddimer, fibrinogen degradation products and other tests 24 hours after admission, and were measured by Roche HP800 automatic biochemical analyzer and Sysmex series automatic blood analyzer.

\section{Controlled Ovarian Hyperstimulation Protocol}

On the $2^{\text {nd }}-3^{\text {rd }}$ days of menstruation, patients were given a longacting gonadotropin-releasing hormone (GnRH) agonist (Diphereline, 3.75 mg; Beaufour-Ipsen, Dreux, France) by subcutaneous injections. Thirty days later, when the FSH level was $<5 \mathrm{IU} / \mathrm{L}$, the LH level was $<3 \mathrm{IU} / \mathrm{L}$, and the antral follicle was nearly 5 $\mathrm{mm}$ in diameter, $\mathrm{COH}$ was initiated. We determined the individualized dosage of gonadotropin ([Gn] GONAL-f; Merck Serono, Darmstadt, Germany) according to the patient's age, BMI, and ovarian reserve. The $\mathrm{Gn}$ dosage was maintained or adjusted according to the follicle growth and serum hormone levels during the course of the drug administration. When one dominant follicle was $\geq 20 \mathrm{~mm}$ in diameter and at least three dominant follicles were $\geq 17 \mathrm{~mm}$ in diameter, a trigger injection of HCG (recombinant human chorionic gonadotropin alfa for injection, Merck Serono) was administered the same night. After 36-37 $\mathrm{h}$ of the trigger injection, we performed transvaginal oocyte retrieval, the luteal phase support is routinely given approximately 14 days after oocyte retrieval. 
Two fresh cleavage embryos or one blastocyst were transferred on day 3 or day 5 after egg retrieval. The transplant was cancelled if patients were deemed at high risk for OHSS, the P level on the day of HCG was $>3 \mathrm{ng} / \mathrm{ml}$, or a uterine effusion was demonstrated.

\section{Statistical Analysis}

All statistical analyses were conducted using IBM SPSS Statistics for Windows, version 26.0. Descriptive variables were expressed as mean and standard deviation (SD) if the data were normally distributed, as median and interquartile range (IQR) if the data were not normally distributed, or as frequency and percentage for nominal data. correlation analysis was used to evaluate the associations between variables of interest and clinical outcomes. Multiple linear regression analysis was used when the outcomes were continuous variables. A bilateral $p$ values $<0.05$ was considered to be significant.

\section{Results}

A total of 424 pregnant patients who developed moderate to severe OHSS after IVF/ICSI treatment were included in this study, and Table 1 summarizes the basic information of the patients. The median recovery time of these patients was 11 days and a detailed distribution of recovery times is shown in Figure 1.

Table 2 Baseline characteristics and cycle outcomes 


\begin{tabular}{|c|c|}
\hline Variables & Measures \\
\hline Age (years) & $30 \rrbracket 27-31 \rrbracket$ \\
\hline BMI (kg/m2) & 20.8凶19.6-22.8\ \\
\hline $\mathrm{AMH} \otimes \mu \mathrm{g} / \mathrm{L} \mathbb{Z}$ & $4.27(2.83-6.33)$ \\
\hline \multicolumn{2}{|l|}{ Type of infertility (n, \%) } \\
\hline Primary infertility & $241(56.8 \%)$ \\
\hline Secondary infertility & $183(43.2 \%)$ \\
\hline \multicolumn{2}{|l|}{ Fertilization method (n, \%) } \\
\hline IVF & $321(75.7 \%)$ \\
\hline ICSI & $103(24.3 \%)$ \\
\hline Gn used duration $(\mathrm{d})$ & $13(12-14)$ \\
\hline Gn used dosage (IU) & $1662.5(1350-2162.5)$ \\
\hline 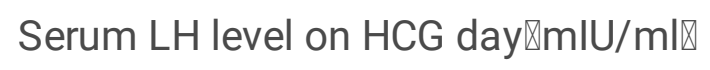 & $0.91(0.5-1.79)$ \\
\hline 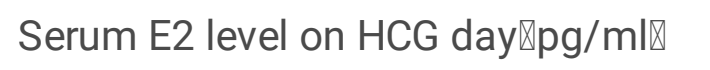 & $3437 ه 2370.5-4769 \rrbracket$ \\
\hline Serum P level on HCG day $₫ \mathrm{ng} / \mathrm{ml} \rrbracket$ & $0.81 \rrbracket 0.51-1.25 \rrbracket$ \\
\hline No. of oocytes retrieved & $13(11-17)$ \\
\hline \multicolumn{2}{|c|}{ Cleavage embryo or blastocyst (Day 3 or Day 5) } \\
\hline Day 3 & $315(74.3 \%)$ \\
\hline Day 5 & $109(25.7 \%)$ \\
\hline recovery time $\mathbb{d} \mathbb{d}$ & 11ه7-15区 \\
\hline
\end{tabular}

Note: BMI Body mass index, AMH antimüllerian hormone,IVF In vitro fertilization, ICSI Intracytoplasmic sperm injection, LH luteinizing hormone, E2 estradiol, P progesterone,

The patient's primary laboratory findings are shown in Table2. Spearman correlation coefficients were calculated between the patient's recovery time and the laboratory indices examined on the day of the patient's admission to the hospital. The levels of leukocytes, hemoglobin, platelets, hematocrit, creatinine, albumin, PT, Fib, TT, D-dimer, and FDP were correlated with the time to healing. Leukocyte, hemoglobin, platelets, hematocrit, creatinine, PT, Fib, D-dimer, and FDP were positively correlated with recovery time. Albumin and TT were negatively correlated with recovery time.

Table 3 Relationship between laboratory findings and recovery time of patients with OHSS 


\begin{tabular}{|c|c|c|c|c|}
\hline \multirow[t]{2}{*}{ Laboratory findings } & \multirow[t]{2}{*}{ Normal range } & \multirow[t]{2}{*}{ Measures } & \multicolumn{2}{|l|}{ Spearman correlation } \\
\hline & & & Correlation coeffcient & $P$ value \\
\hline Leukocyte (10^9/L) & $3.5-9.5$ & 12.97凶10.6-15.91》 & 0.401 & $<0.001$ \\
\hline Hemoglobin (g/L) & $115-150$ & 143.65囚135.25-153囚 & 0.494 & $<0.001$ \\
\hline Platelets $\left(10^{\wedge} 9 / \mathrm{L}\right)$ & $125-350$ & $340.5 \rrbracket 293-386 \rrbracket$ & 0.245 & $<0.001$ \\
\hline Hematocrit (L/L) & $0.35-0.45$ & $0.429 \rrbracket 0.402-0.458 \rrbracket$ & 0.478 & $<0.001$ \\
\hline Creatinine (mmol/L) & $20-115$ & $58.75 \otimes 52-66.18 \rrbracket$ & 0.352 & $<0.001$ \\
\hline $\operatorname{ALT}(\mathrm{U} / \mathrm{L})$ & $0-40$ & $26 \rrbracket 15.25-41 \rrbracket$ & -0.027 & 0.577 \\
\hline AST (U/L) & $0-58$ & $22.5 \rrbracket 17-32.75 \rrbracket$ & -0.071 & 0.146 \\
\hline Albumin (g/L) & $35-55$ & $37.5 \rrbracket 34.9-40.1 \rrbracket$ & -0.202 & $<0.001$ \\
\hline PT囚s》 & $8.8-13.6$ & 10.3凶9.9-10.8凶 & 0.151 & 0.002 \\
\hline APTT $(s \rrbracket$ & $26-40$ & $28.3 \rrbracket 26.6-30.2 \rrbracket$ & -0.075 & 0.125 \\
\hline $\mathrm{Fib}(\mathrm{g} / \mathrm{L})$ & $2-4$ & $4.65 \rrbracket 4.3-5.32 \rrbracket$ & 0.175 & $<0.001$ \\
\hline TT (s) & $10-18$ & 12.6ه12.1-13.1》 & -0.113 & 0.020 \\
\hline D-dimer (mg/L) & $0-0.3$ & $0.70 \rrbracket 0.52-0.95 \rrbracket$ & 0.136 & 0.005 \\
\hline $\mathrm{FDP}(\mathrm{mg} / \mathrm{L})$ & $0-5$ & $7.23 \rrbracket 5.19-10.54 \rrbracket$ & 0.114 & 0.018 \\
\hline
\end{tabular}

Note: ALT Alanine aminotransferase, AST Aspartate transaminase, PT Prothrombin time, APTT Activated partial thromboplastin time, TT Thrombin tim, Fib Fibrinogen, FDP Fibrinogen degradation products

All information with $\mathrm{p}<0.05$ in the above-mentioned correlation analysis and the high-risk factors affecting OHSS reported in the literature are included in the multiple linear regression. The results showed that PCOS, hemoglobin, platelets, albumin, and Fib significantly influenced the patient's recovery time $(p=$ $0.023, p<0.001, p=0.007, p<0.001, p=0.019$; respectively) (Table 3). Moreover, scatter diagrams were used to clearly describe the relationship between the recovery time and hemoglobin, platelets, albumin, and fib (Figure 2). The remaining indicators were not highly correlated with patient recovery time.

Table 4 Multiple linear regression analysis of factors affecting recovery time 


\begin{tabular}{|c|c|c|c|c|c|c|c|}
\hline \multirow[t]{2}{*}{ Indexes } & \multicolumn{2}{|c|}{$\begin{array}{l}\text { Unstandardized } \\
\text { coefficients }\end{array}$} & \multirow{2}{*}{$\begin{array}{l}\text { Standardized } \\
\text { coefficients } \\
\beta\end{array}$} & \multirow[t]{2}{*}{$\begin{array}{l}\mathrm{t} \\
\text { value }\end{array}$} & \multirow[t]{2}{*}{$\begin{array}{l}P \\
\text { value }\end{array}$} & \multicolumn{2}{|l|}{$\begin{array}{l}\text { Collinearity } \\
\text { statistics }\end{array}$} \\
\hline & B & Std.Error & & & & Tolerance & VIF \\
\hline Age (year) & -0.006 & 0.089 & -0.003 & -0.066 & 0.947 & 0.939 & 1.065 \\
\hline BMI (kg/m2) & -0.130 & 0.104 & -0.055 & -1.244 & 0.214 & 0.901 & 1.110 \\
\hline AMH $\rrbracket \mu \mathrm{g} / \mathrm{L} \otimes$ & -0.086 & 0.082 & -0.049 & -1.051 & 0.294 & 0.805 & 1.242 \\
\hline PCOS & -1.988 & 0.871 & -0.105 & -2.281 & 0.023 & 0.821 & 1.218 \\
\hline 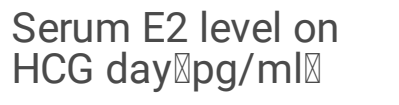 & 0.000 & 0.000 & 0.005 & 0.102 & 0.919 & 0.877 & 1.141 \\
\hline $\begin{array}{l}\text { No. of oocytes } \\
\text { retrieved }\end{array}$ & 0.073 & 0.059 & 0.061 & 1.245 & 0.214 & 0.733 & 1.365 \\
\hline $\begin{array}{l}\text { Cleavage embryo or } \\
\text { blastocyst }\end{array}$ & -0.314 & 0.582 & -0.028 & -0.540 & 0.589 & 0.669 & 1.495 \\
\hline $\begin{array}{l}\text { Clinical pregnancy } \\
\text { (singleton or } \\
\text { multiple) }\end{array}$ & -0.190 & 0.607 & -0.015 & -0.312 & 0.755 & 0.779 & 1.284 \\
\hline Leukocyte (10^9/L) & 0.077 & 0.079 & 0.057 & 0.981 & 0.327 & 0.523 & 1.910 \\
\hline Hemoglobin (g/L) & 0.146 & 0.024 & 0.349 & 6.213 & $<.001$ & 0.552 & 1.811 \\
\hline Platelets $\left(10^{\wedge} 9 / \mathrm{L}\right)$ & 0.010 & 0.004 & 0.129 & 2.690 & 0.007 & 0.752 & 1.329 \\
\hline Hematocrit \L/L】 & 0.039 & 0.069 & 0.025 & 0.570 & 0.569 & 0.939 & 1.065 \\
\hline Creatinine $₫ \mathrm{mmol} / \mathrm{L} \rrbracket$ & 0.015 & 0.026 & 0.029 & 0.562 & 0.575 & 0.656 & 1.523 \\
\hline Albumin (g/L) & -0.239 & 0.067 & -0.154 & -3.587 & $<.001$ & 0.944 & 1.060 \\
\hline PT』s》 & 0.046 & 0.052 & 0.037 & 0.880 & 0.379 & 0.981 & 1.019 \\
\hline Fibrinogen $(\mathrm{g} / \mathrm{L})$ & 0.835 & 0.356 & 0.113 & 2.348 & 0.019 & 0.755 & 1.324 \\
\hline $\mathrm{TT} \rrbracket s \rrbracket$ & -0.227 & 0.269 & -0.043 & -0.843 & 0.400 & 0.662 & 1.510 \\
\hline D-dimer》mg/L】 & 0.537 & 0.553 & 0.056 & 0.969 & 0.333 & 0.517 & 1.934 \\
\hline $\begin{array}{l}\text { Fibrinogen } \\
\text { degradation } \\
\text { products } \otimes g / L \otimes\end{array}$ & -0.032 & 0.071 & -0.024 & -0.442 & 0.659 & 0.587 & 1.705 \\
\hline
\end{tabular}

\section{Discussion}

OHSS is a major iatrogenic complication that arises during the processes of assisted reproductive technology (ART), affecting $0.5 \%$ to $2 \%$ of IVF cycles. There are two distinct types of OHSS. Early onset 
OHSS occurs in response to HCG trigger within 7 days of ovulation and late onset OHSS which is caused by the rising HCG hormone levels produced by the placenta in conception cycles [10]. The present study focused on the recovery time of OHSS requiring hospitalization in conceived women.

To date, there is no universally accepted definition of OHSS recovery. The definition in our research is based on previous study [19]. Patients were discharged from hospital when morning hematocrit was $<40 \%$ and no obvious clinically relevant symptoms were existed, such as abdominal distension, abdominal pain and shortness of breath. Meanwhile, ultrasound indicated little pleural or abdominal effusion and biochemical abnormalities return to normal were required. The median recovery time in our study population was 11 days. This is much longer than that reported in the literature, partly due to the particular population and our strict release criteria.

Currently studies revealed that the incidence of OHSS was associated with numerous clinical and laboratory parameters. The concentration of E2 on the day of HCG $>4500 \mathrm{pg} / \mathrm{ml}$ and the number of oocytes retrieved > 15 are commonly proposed to be risk factors for OHSS [3]. However, our results showed that the median concentration of E2 on the day of HCG was $3437 \mathrm{pg} / \mathrm{ml}$, and the median number of oocytes retrieved was 13. This is consistent with Wiser and colleague's study because freeze-all strategy was carried out in women at high risk of OHSS after oocytes retrieved [20]. In addition, the concentration of E2 on the day of HCG is not the main reason for the late onset OHSS and pregnancy remains the main cause [21]. Despite scholars proposed multiple pregnancy as a predictive factor for recovery time of OHSS, our research found no differences between singleton and multiple pregnancy patients.

As demonstrated in Table 3, the presence of PCOS was negatively correlated with recovery time of pregnant OHSS patients, which is in accordance with the result of Kazem Nouri et al [19]. PCOS appeared to be the major predisposing factor for OHSS in a large number of studies [2]. The explanation might be that PCOS cases are known to produce three times more follicles and oocytes than normal ovulation patients when stimulated according to similar protocols [6]. However, the reason for the prolonged recovery time of PCOS patients remains unknown. Vascular endothelial growth factor (VEGF) is thought to be the major mediator of OHSS [22]. An increased expression of VEGF mRNA in women with PCOS has been reported, and this may be responsible for the prolonged recovery time [23]. Nevertheless, this is completely unproven. Further investigations are necessary to confirm this hypothesis. We suggest that PCOS patients should administer more stringent embryo transfer criteria or undergo whole embryo cryopreservation.

The second predictive factor for recovery time is serum albumin. Hypoalbuminemia was associated with significantly longer recovery time. The mechanisms beneath the potential effect of serum albumin on OHSS is unknown. Some studies suggest that the binding properties of albumin are beneficial in neutralizing vascular permeability mediators [24]. Other studies have shown that serum albumin could maintain the intravascular volume in the event of capillary leakage thus avoiding hypovolemia and hemoconcentration [25]. Therefore, the level of serum albumin may reflect the severity of OHSS. 
Studies demonstrated that the levels of leukocyte, platelets, Hematocrit, Fib, D-dimer and FDP of OHSS patients were higher in routine laboratory tests [26]. As shown in Table 2, this is consistent with our study. Moreover, in multiple linear regression analysis, hemoglobin, platelets and Fib were positively correlated with recovery time, and the difference was statistically significant. There are two reasons for the changes of these blood-related parameters: stress and hemoconcentration [27]. OHSS is a potentially lethal disease, the pathophysiological hallmark of which is massive extravascular exudate accumulation combined with profound intravascular volume depletion and hemoconcentration [28]. The degree of hemoconcentration seems to have the best correlation with severity of OHSS [29]. Hemoglobin, platelets and Fib are the earliest and most sensitive indicators of changes in the blood, and are also ideal predictors for recovery time of OHSS.

It seems that elevation in circulating estrogens during ovulation induction cause a shift in the haemostatic balance in the direction of a procoagulable state. FIB is a conventional coagulation indicator that indicates activation of the coagulation system. Coagulation causes increased FIB consumption and promotes FIB synthesis in the body resulting in increased plasma FIB levels. Thrombosis is the most serious complication of OHSS, leading to dysfunction of coagulation and fibrinolysis in vivo. Thrombosis causes secondary hyperfibrinolysis, and plasma D-dimer and FDP are degradation products of fibrinogen, so the levels of D-dimer and FDP will increase rapidly. Therefore, monitoring plasma FIB囚D-dimer and FDP can prompt clinical correction of hypercoagulable blood concentration. Heparin ameliorates the risk of thrombotic complications associated with OHSS and has become the recommended treatment protocols [30]. VEGF plays a leading role in increasing vascular permeability, and a $5 \mathrm{kDa}$ heparin fragment can inhibit VEGF-A-mediated angiogenesis [31]. TF is also important in angiogenesis because it enhances VEGF-A expression, and heparin reduces VEGF expression by inhibiting TF release [32].

This is the first study to comprehensively assess the coagulation function and recovery time of conceived women with moderate to severe OHSS. As a result of OHSS-specific symptoms and costs due to treatment program, the decreased life quality and economic loss are inevitable. Therefore, the recovery

time should be a clinically important parameter and our study is of great value. Simultaneously, our study has some limitations. We conducted this retrospective study without considering all confounding factors. Only patients receiving particular ovulation induction program were included in our study. Moreover, the differences in patients' dietary habits during hospitalization have a certain impact on the recovery time of OHSS.

\section{Conclusion}

In general, the existence of PCOS囚the levels of hemoglobin, platelets, albumin, and Fib may contribute to the prognostic evaluation of OHSS. In consideration of the particular study population and limitations, large scale, multicenter, prospective studies are necessary to confirm our results.

\section{Abbreviations}


OHSS: Ovarian hyperstimulation syndrome; IVF: In vitro fertilization; ICSI: Intracytoplasmic sperm injection; ALT: Alanine transaminase; AST: Aspartate transaminase; PT: Prothrombin time; APTT: Activated partial thromboplastin time; TT:Thrombin time; Fib:Fibrinogen; FDP:Fibrinogen degradation products; $\mathrm{COH}$ : Controlled ovarian hyperstimulation; BMI: Body mass index; PCOS: Polycystic ovary syndrome; AMH: Antimüllerian hormone; E2 estradiol; LH luteinizing hormone; hCG: Human chorionic gonadotropin; VEGF: Vascular endothelial growth factor; GnRH: Gonadotropin-releasing hormone; SD: Standard deviation; IQR: Interquartile range; ART: Assisted reproductive technology.

\section{Declarations}

\section{Acknowledgements}

We would like to thank the medical staff and patients in the First Affifiliated Hospital of Zhengzhou University for recording the data and cooperating with the treatment.

\section{Authors' contributions}

Kai Huang and Jun Zhai have designed the research and guided writing. Ying Shi collected the data, analyzed the data. Kai Huang and Ying Shi drafted the manuscript. Gezi Chen helped to collect and analyze data. Hao Shi contributed to the data analysis. All authors read and approved the final manuscript.

\section{Funding}

This study was supported by the National Natural Science Foundation of China (No. 81701448 and No. 82071649).

\section{Availability of data and materials}

All data supporting the conclusion of this article are included.

\section{Declarations}

\section{Ethics approval and consent to participate}

The study has received approval and was carried out in accordance with the approved guidelines from the Zhengzhou University Research Ethics Board.

\section{Consent for publication}

Not applicable.

\section{Competing interests}

The authors declare that they have no competing interests. 


\section{Author details}

${ }^{1}$ Center for Reproductive Medicine, Henan Key Laboratory of Reproduction and Genetics, Henan Provincial Obstetrical and Gynecological Diseases (Reproductive Medicine) Clinical Research Center, Henan Engineering Laboratory of Preimplantation Genetic Diagnosis and Screening, The First Affiliated Hospital of Zhengzhou University, Zhengzhou, China. ${ }^{2}$ Department of Obstetrics, The First Affiliated Hospital of Zhengzhou University, Zhengzhou, China.

\section{References}

1. ASRM. Prevention and treatment of moderate and severe ovarian hyperstimulation syndrome: a guideline. Fertil Steril, 2016;106(7):1634-1647.

2. Luke B, Brown MB, Morbeck DE, Hudson SB. Factors associated with ovarian hyperstimulation syndrome (OHSS) and its effect on assisted reproductive technology (ART) treatment and outcome. Fertil Steril, 2010;94(4):1399-1404.

3. Sousa M, Cunha M, Teixeira da Silva J, Oliveira C, Silva J, Viana P, Barros A. Ovarian hyperstimulation syndrome: a clinical report on 4894 consecutive ART treatment cycles. Reprod Biol Endocrinol, 2015;13:66.

4. Fauser BC, Diedrich K, Devroey P; Evian Annual Reproduction Workshop Group 2007. Predictors of ovarian response: progress towards individualized treatment in ovulation induction and ovarian stimulation. Hum Reprod Update, 2008;14(1):1-14.

5. Courbiere B, Oborski V, Braunstein D, Desparoir A, Noizet A, Gamerre M. Obstetric outcome of women with in vitro fertilization pregnancies hospitalized for ovarian hyperstimulation syndrome: a casecontrol study. Fertil Steril, 2011;95(5):1629-1632.

6. Delvigne A, Rozenberg S. Epidemiology and prevention of ovarian hyperstimulation syndrome (OHSS): a review. Hum Reprod Update, 2002;8(6):559-577.

7. Nakhuda GS, Chu MC, Wang JG, Sauer MV, Lobo RA. Elevated serum müllerian-inhibiting substance may be a marker for ovarian hyperstimulation syndrome in normal women undergoing in vitro fertilization. Fertility and sterility, 2006;85(5):1541-1543.

8. Ocal P, Sahmay S, Cetin M, Irez T, Guralp O, Cepni I. Serum anti-Mullerian hormone and antral follicle count as predictive markers of OHSS in ART cycles. J Assist Reprod Genet, 2011;28(12):1197-1203.

9. Ashrafi M, Bahmanabadi A, Akhond MR, Arabipoor A. Predictive factors of early moderate/severe ovarian hyperstimulation syndrome in non-polycystic ovarian syndrome patients: a statistical model. Arch Gynecol Obstet, 2015;292(5):1145-1152.

10. Mathur RS, Akande AV, Keay SD, Hunt LP, Jenkins JM. Distinction between early and late ovarian hyperstimulation syndrome. Fertil Steril, 2000;73(5):901-907.

11. Steward RG, Lan L, Shah AA, Yeh JS, Price TM, Goldfarb JM, Muasher SJ. Oocyte number as a predictor for ovarian hyperstimulation syndrome and live birth: an analysis of 256,381 in vitro fertilization cycles. Fertility and Sterility, 2014;101(4):967-973. 
12. Enskog A, Henriksson M, Unander M, Nilsson L, Brannstrom M. Prospective study of the clinical and laboratory parameters of patients in whom ovarian hyperstimulation syndrome developed during controlled ovarian hyperstimulation for in vitro fertilization. Fertil Steril, 1999;71(5):808-814.

13. Delvigne A, Dubois M, Battheu B, Bassil S, Meuleman C, De Sutter P, Rodesch C, Janssens P, Remacle $\mathrm{P}$, Gordts S. The ovarian hyperstimulation syndrome in in-vitro fertilization: a Belgian multicentric study. II. Multiple discriminant analysis for risk prediction. Hum Reprod, 1993;8(9):1361-1366.

14. Aboulghar MA, Mansour RT. Ovarian hyperstimulation syndrome: classifications and critical analysis of preventive measures. Hum Reprod Update, 2003;9(3):275-289.

15. McClure N, Healy DL, Rogers PA, Sullivan J, Beaton L, Haning RV Jr, Connolly DT, Robertson DM. Vascular endothelial growth factor as capillary permeability agent in ovarian hyperstimulation syndrome. Lancet, 1994;344(8917):235-236.

16. Soares SR, Gómez R, Simón C, García-Velasco JA, Pellicer A. Targeting the vascular endothelial growth factor system to prevent ovarian hyperstimulation syndrome. Hum Reprod Update, 2008;14(4):321-333.

17. Geva E, Jaffe RB. Role of vascular endothelial growth factor in ovarian physiology and pathology. Fertil Steril, 2000;74(3):429-438.

18. Choux C, Barberet J, Ginod P, Cottenet J, Bruno C, Benzénine E, Sagot P, Fauque P. Severe ovarian hyperstimulation syndrome modifies early maternal serum beta-human chorionic gonadotropin kinetics, but obstetrical and neonatal outcomes are not impacted. Fertil Steril, 2017;108(4):650-658.

19. Nouri K, Tempfer CB, Lenart C, Windischbauer L, Walch K, Promberger R, Ott J. Predictive factors for recovery time in patients suffering from severe OHSS. Reprod Biol Endocrinol, 2014;12(59).

20. Wiser A, Levron J, Kreizer D, Achiron R, Shrim A, Schiff E, Dor J, Shulman A. Outcome of pregnancies complicated by severe ovarian hyperstimulation syndrome (OHSS): a follow-up beyond the second trimester. Hum Reprod, 2005;20(4):910-914.

21. Salama KM, Abo Ragab HM, El Sherbiny MF, Morsi AA, Souidan II. Sequential E2 levels not ovarian maximal diameter estimates were correlated with outcome of cetrotide therapy for management of women at high-risk of ovarian hyperstimulation syndrome: a randomized controlled study. BMC Womens Health, 2017;17(1):108.

22. Fiedler K, Ezcurra D. Predicting and preventing ovarian hyperstimulation syndrome (OHSS): the need for individualized not standardized treatment. Reprod Biol Endocrinol, 2012;10(1):32.

23. Kamat BR, Brown LF, Manseau EJ, Senger DR, Dvorak HF. Expression of vascular permeability factor/vascular endothelial growth factor by human granulosa and theca lutein cells. Role in corpus luteum development. Am J Pathol, 1995;146(1):157-165.

24. Youssef MA, Al-Inany HG, Evers JL, Aboulghar M. Intra-venous fluids for the prevention of severe ovarian hyperstimulation syndrome. Cochrane Database Syst Rev, 2011;2(2):CD001302.

25. Nastri CO, Teixeira DM, Moroni RM, Leitão VM, Martins WP. Ovarian hyperstimulation syndrome: pathophysiology, staging, prediction and prevention. Ultrasound Obstet Gynecol, 2015;45(4):377-393. 
26. Levin I, Gamzu R, Hasson Y, Lessing JB, Amit A, Shapira I, Berliner S, Almog B. Increased erythrocyte aggregation in ovarian hyperstimulation syndrome: a possible contributing factor in the pathophysiology of this disease. Hum Reprod, 2004;19(5):1076-1080.

27. Navot D, Bergh PA, Laufer N. Ovarian hyperstimulation syndrome in novel reproductive technologies: prevention and treatment. Fertility and Sterility, 1992;58(2):249-261.

28. Schenker JG, Weinstein D. Ovarian Hyperstimulation Syndrome: A Current Survey. Fertility and Sterility, 1978;30(3):255-268.

29. Borenstein R, Elhalah U, Lunenfeld B, Schwartz ZS. Severe ovarian hyperstimulation syndrome: a reevaluated therapeutic approach. Fertility and Sterility, 1989;51(5):791-795.

30. Dourron NE, Williams DB. Prevention and treatment of ovarian hyperstimulation syndrome. Semin Reprod Endocrinol, 1996;14(4):355-365.

31. K, Norrby. $2.5 \mathrm{kDa}$ and $5.0 \mathrm{kDa}$ heparin fragments specifically inhibit microvessel sprouting and network formation in VEGF165-mediated mammalian angiogenesis. Int J Exp Pathol, 2000;81(3):191-198.

32. Bobek V, Kovarík J. Antitumor and antimetastatic effect of warfarin and heparins. Biomed Pharmacother, 2004;58(4):213-219.

\section{Figures}

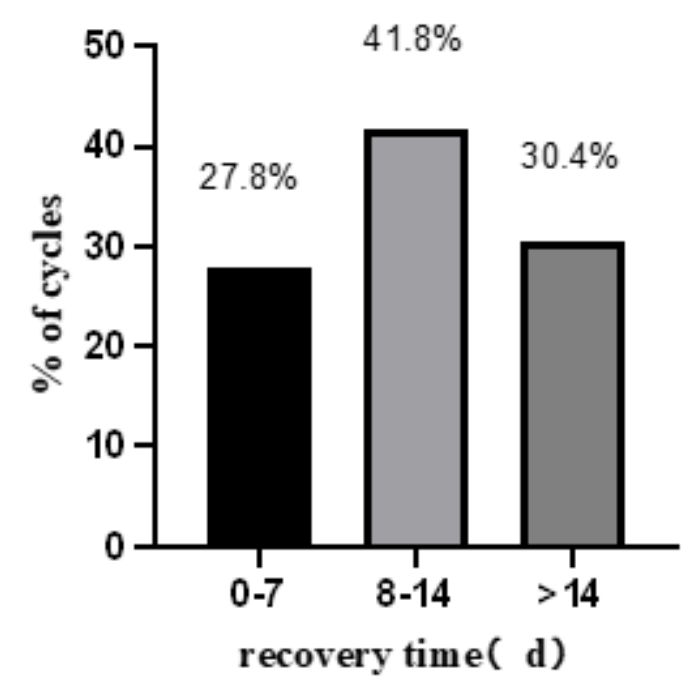

\section{Figure 1}

The recovery time distribution of the 424 OHSS patients 
A

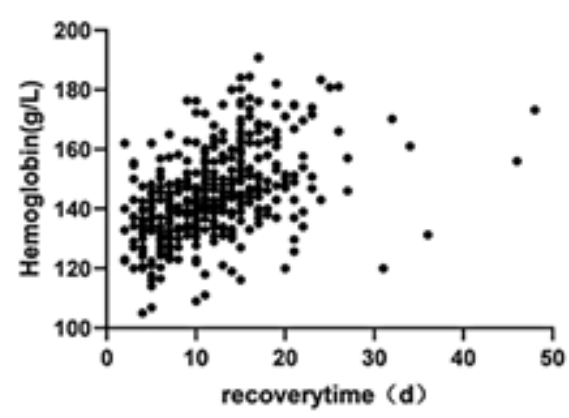

C

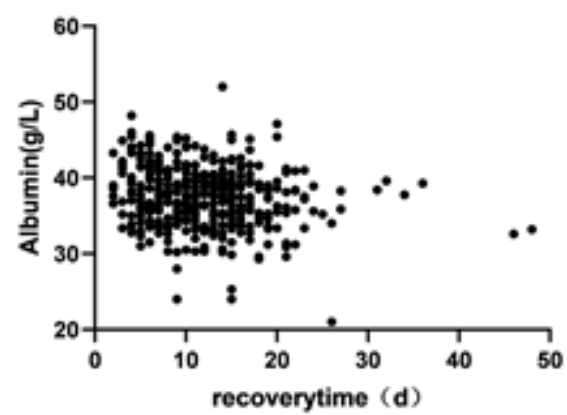

B

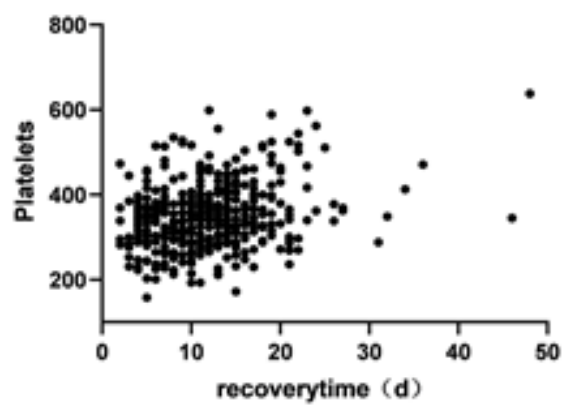

D

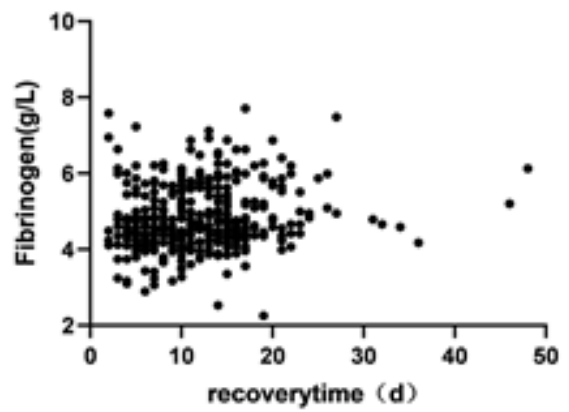

Figure 2

Relationship between the recovery time and hemoglobin, platelets, albumin, and Fib 